\title{
Evaluating Cytotoxicity of Hyaluronate Targeted Solid Lipid Nanoparticles of Etoposide on SK-OV-3 Cells
}

\author{
Parviz Mohammadi Ghalaei, ${ }^{1}$ Jaleh Varshosaz, ${ }^{1}$ and Hojatollah Sadeghi Aliabadi ${ }^{2}$ \\ ${ }^{1}$ Department of Pharmaceutics, School of Pharmacy and Novel Drug Delivery Systems Research Centre, \\ Isfahan University of Medical Sciences, P.O. Box 81745-359, Isfahan 81746-73461, Iran \\ ${ }^{2}$ Department of Pharmaceutical Biotechnology, School of Pharmacy and Pharmaceutical Sciences, \\ Isfahan University of Medical Sciences, Isfahan 81746-73461, Iran \\ Correspondence should be addressed to Jaleh Varshosaz; varshosaz@pharm.mui.ac.ir
}

Received 1 January 2014; Revised 28 March 2014; Accepted 28 March 2014; Published 24 April 2014

Academic Editor: Sami M. Nazzal

Copyright (C) 2014 Parviz Mohammadi Ghalaei et al. This is an open access article distributed under the Creative Commons Attribution License, which permits unrestricted use, distribution, and reproduction in any medium, provided the original work is properly cited.

\begin{abstract}
The epithelial ovarian carcinoma is one of the most fatal gynecological cancers. Etoposide is used in treating platinum-resistant ovarian cancer. Sodium hyaluronate is a substance that binds to the $\mathrm{CD}_{44}$ receptors overexpressed in SK-OV-3 cells of epithelial ovarian carcinoma. The aim of the present work was to study the cytotoxicity effect of hyaluronate targeted solid lipid nanoparticles (SLNs) of etoposide on SK-OV-3 cells. The cytotoxicity of the targeted and nontargeted SLNs of etoposide was compared to free drug on the SK-OV-3 cells by MTT assay method. The cellular uptake of the targeted and nontargeted nanoparticles containing sodium fluorescein was also studied. The difference of cell vitality between nontargeted nanoparticles and also targeted nanoparticles with free drug was significant. Targeted nanoparticles also caused more toxicity than nontargeted nanoparticles $(P<0.05)$. After 4 hours of incubating, the fluorescence was remarkably higher in the cells treated by targeted SLNs rather than nontargeted ones, and there was no observable fluorescence in cells incubated with pure sodium fluorescein. Hyaluronate targeted SLNs containing etoposide increased the cytotoxicity of etoposide on SK-OV-3 cells which may be a worthwhile potential method for reducing the prescribed dose and systemic side effects of this drug in epithelial ovarian carcinoma.
\end{abstract}

\section{Introduction}

The epithelial ovarian carcinoma is one of the most fatal gynecological cancers across the globe. In spite of early recovery by surgical and chemotherapy treatments, the 5year survival rate for the patients is only 13 percent. The database GLOBCAN related to the World Health Organization (WHO) has reported incidence of about 192000 cases in the world, in the year of 2000. 6000 cases of the mentioned cases have occurred in the UK, and 21000 cases in the U.S. For treating the disease, the tumor will be removed by surgical procedures and then chemotherapy would be started with platinum-based chemotherapy (cisplatin and carboplatin), which treating regime includes cisplatin and carboplatin with the drugs such as paclitaxel, docetaxel, cyclophosphamide, and doxorubicin. In some of the patients, the disease relapses after 6 months of chemotherapy; this condition is defined as platinum resistant, in which treatment would be continued with drugs such as topotecan and etoposide [1].

Etoposide, as other chemotherapy agents, has many side effects such as bone marrow suppression, granulocytopenia, thrombocytopenia [2], mucositis, moderate to severe esophagitis, hepatotoxicity, metabolic acidosis, and anemia [3].

The complications of anticancer drugs have caused scientists to try two approaches to solve the problem: developing new drugs with fewer side effects and application of new drug delivery systems with high specificity to cancerous tissues; the second approach has lower costs and more attention nowadays. Solid lipid nanoparticles (SLNs) are one of the most important nanosized drug delivery systems that were introduced about two decades ago [4].

SLNs that are often considered for intravenous application are colloidal submicron carriers sized 50 to $1000 \mathrm{~nm}$ 
and composed of solid lipids dispersed in water or surfactant aqueous solution. These nanoparticles have particular features like small size, high surface area, and high loading of drug that makes them potent and beneficial carriers for improving drug efficacy $[5,6]$. SLNs are similar to o/w emulsions used for total parenteral nutrition; the difference is that emulsion liquid lipid has been replaced with a solid lipid. SLNs have advantages such as controlled drug release in considered site, excellent biocompatibility, increase in drug stability, high drug content, easy industrialization and sterilization, better control of drug release kinetics, high bioavailability for bioactive drugs, chemical protection of sensitive drugs, easier producing rather than biopolymeric nanoparticles, producible by common emulsification methods, long-time stability, and various applications $[4,7,8]$.

For parenteral administration, SLN dispersions must be sterile. SLNs with appropriately small particle size less than $200 \mathrm{~nm}$ can be sterilized using filtration. Autoclaving the finished dispersion is not practical as the lipids melt at sterilizing temperatures and the molten lipid droplets coalesce. Therefore just aseptic manufacturing processes following sterilization of the starting materials by gamma irradiation of the final dispersion or exposure to ethylene oxide (EO) gas are applicable for their sterilization. Bacterial endotoxins in raw materials need to be monitored, especially when raw materials are of natural origin. It may be possible to lyophilize the SLN dispersions, and this lyophile can be irradiated or exposed to EO.

SLNs are used in transdermal applications, as gene vector carriers, for topical uses, as cosmeceuticals, as targeted carriers of anticancer drugs to solid tumors, in breast cancer and lymph node metastases and in antitubercular chemotherapy.

So far successful studies have been performed upon nanoparti-cles containing etoposide. For example, the study of Yadav et al. [9] was performed in the survey of poly(lacticcoglycolic acid)-monomethoxy-poly(polyethylene glycol) and poly(lactic-coglycolic acid)-Pluronic block copolymers and the study of Reddy et al. [10] on nanoparticles produced by tripalmitin could be mentioned.

Hyaluronan (Figure 1), available in the market as sodium hyaluronate (HA), is a high molecular weight glycosaminoglycan present in extracellular matrix and is necessary for cellular growth and structural stability of organs and tissue structure.

HA regulates cell proliferation and movements by interacting with $\mathrm{CD}_{44}$ receptors and receptor for $\mathrm{HA}$ mediated motility (RHAMM). Because of overexpression of $\mathrm{CD}_{44}$ receptors by cancer cells, interfering in $\mathrm{CD}_{44}$ - $\mathrm{HA}$ interaction by targeting drugs at $\mathrm{CD}_{44}$ is an effective strategy to treat cancers. HA bound to nanoparticles, in addition to its targeting role, may act as a protecting agent of nanoparticles against body phagocytosis system [11-13]. The mentioned method has been used to deliver agents such as doxorubicin [14], epirubicin [15], paclitaxel [16], mitomycin C [17], SiRNA [18], and DNA [19].

To our knowledge there is not any report on the application of the hyaluronate targeted SLNs in drug delivery of etoposide in SK-OV-3 cells although there are some studies on the hyaluronate targeted SLNs. This study alongside

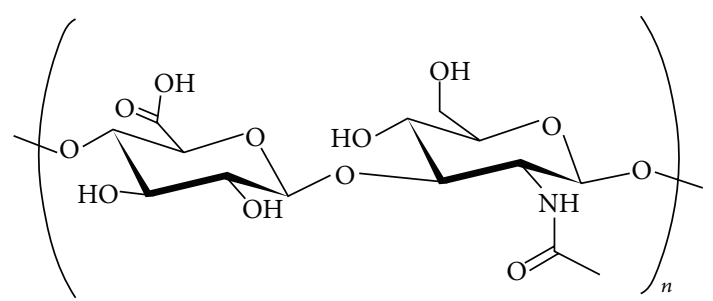

FIGURE 1: Chemical structure of hyaluronan: polymeric repeat of $D$ glucuronic acid and $\mathrm{N}$-acetylglucosamine.

with thousands of similar ones could help to introduce new clinically applicable drug delivery systems with appropriate physicochemical properties, successful targeting, and enhanced cytotoxicity in the future. This study was performed in order to evaluate cytotoxicity of HA targeted SLNs containing etoposide, prepared and optimized in our previous study [20] in SK-OV-3 cells.

\section{Materials and Methods}

2.1. Materials. Stearylamine (SA), dodecylamine (DDA), cetyl alcohol, dialysis bags with molecular weight cut-off of $12400 \mathrm{Da}$, and thiazolyl blue tetrazolium bromide (MTT) were from Sigma-Aldrich Company (US). Acetone, dichloromethane, and Tween 80 were from Merck Chemical Company (Germany). RPMI 1640 culture medium, penicillinstreptomycin, and fetal bovine serum were from PAA Company, Austria. Etoposide was a gift from Nippon Kayaku Co, Ltd. (Tokyo, Japan). Sodium hyaluronate $(\mathrm{Mw}=6,400 \mathrm{Da})$ was from Lifecore Biomedical (US) and SK-OV-3 cells were from Pasteur Institute (Iran).

2.2. Preparing Nanoparticles. SLNs were produced by emulsification-solvent evaporation method. According to the results of our previous study [20], the lipid phase including $30 \mathrm{mg}$ etoposide, $30 \mathrm{mg}$ cetyl alcohol, and $30 \mathrm{mg}$ SA was dissolved in $1.8 \mathrm{~mL}$ of $1: 1$ mixture of acetonedichloromethane. Then the mentioned solution was added during 3 minutes to the $18 \mathrm{~mL}$ of Tween 80 solution (1\% w/v) in deionized water, while stirring in $1200 \mathrm{rpm}$. Ultimately, produced nanoemulsion was stirred in $600 \mathrm{rpm}$ in room temperature for 75 minutes to evaporate the solution [21]. The blank nanoparticles were produced by the same method but without etoposide.

2.3. Physical Binding of HA to the SLNs Surface. After 15 minutes of adding organic phase to aqueous phase, HA dissolved in deionized water containing Tween $80(1 \% \mathrm{w} / \mathrm{v})$ was added to nanoparticles mixture during 5 minutes, while stirring at $600 \mathrm{rpm}$, in order to produce targeted nanoparticles [22].

Nonbound HA was separated from nanoparticles mixture by dialyzing versus $100 \mathrm{~mL}$ deionized water containing Tween $80(1 \% \mathrm{w} / \mathrm{v})$ using dialysis bag with molecular weight cut-off of $12,400 \mathrm{Da}$ for 40 minutes so that the deionized water containing Tween $80(1 \% \mathrm{w} / \mathrm{v})$ was replaced every 10 minutes. To determine the amount of HA bounded to 
SLNs after separation of unbound HA, some part of the targeted nanoparticles mixture was dried under vacuum and subjected to elemental analysis (CHN) (CHNS-932, Leco, USA) and, by subtracting the total amount of HA from gaining value, the amount of HA bound on the SLNs surface was calculated.

2.4. Measuring Particle Size, Polydispersity Index, and Zeta Potential. The particle size, polydispersity index, and zeta potential of nanoparticles were measured by a Zetasizer (Zetasizer 3000; Malvern Instruments, Malvern, UK), after $1: 10$ diluting the samples with deionized water.

2.5. Determining Drug Loading and Release. The loading efficiency percent was determined by centrifugation (Eppendorf 5430 centrifuge, Germany). The dispersion of nanoparticles was poured in centrifugal filter tubes (Amicon Ultra, Ireland) with a $10 \mathrm{kDa}$ molecular weight cutoff to separate the aqueous medium [23]. The concentration of free etoposide in the filtrate was determined by measuring its absorption in $276.4 \mathrm{~nm}$ (UV-VIS spectrophotometer, Shimadzu Scientific Instruments, Japan) and converting the absorbance to concentration using the calibration equation of etoposide in aqueous phase containing $1 \% \mathrm{w} / \mathrm{v}$ of Tween 80 . The amount of encapsulated drug was computed indirectly by calculating the difference between the total amounts of drug used in preparation of nanoparticles and the free drug. Ultimately, loading efficiency percent was computed by the following equation:

\section{Loding efficiency percent}

$$
=\frac{(\text { total drug weight }- \text { free drug weight })}{\text { total drug weight }} \times 100 \text {. }
$$

Drug release profiles from the NPLs were determined in phosphate buffer saline (PBS, $0.01 \mathrm{M}, \mathrm{pH} 7.4$ containing $1 \%$ w/v Tween 80 ) at $37^{\circ} \mathrm{C}$. A total of $2 \mathrm{~mL}$ of NPLs suspension was placed in dialysis bag with molecular weight cut-off of $12,400 \mathrm{Da}$ and suspended in a beaker containing $50 \mathrm{~mL}$ of PBS on a magnetic stirrer with a speed of $200 \mathrm{rpm}$. Samples were withdrawn periodically and replaced with the same volume of PBS at the same temperature. The content of etoposide in the samples was determined spectrophotometrically at $268.7 \mathrm{~nm}$.

2.6. MTT Colorimetric Cytotoxicity Assay. To determine cell proliferation, an MTT assay was carried out. A total of $180 \mu \mathrm{L}$ of the cell suspension $\left(5 \times 10^{4}\right.$ cells $\left./ \mathrm{mL}\right)$ were placed in each well of a 96-well plate except for one row for blank that was filled by an equal amount of medium. After a $24 \mathrm{~h}$ period of incubation at $37^{\circ} \mathrm{C}$ in a $\mathrm{CO}_{2}$ incubator with $5 \% \mathrm{CO}_{2}$ and $95 \%$ humidity, all 4 wells of cells were treated with $20 \mu \mathrm{L}$ of one of the concentrations of etoposide as much as $0.475,0.95$, 1.9 , and $3.8 \mu \mathrm{M}$ of etoposide. The $\mathrm{IC}_{50}$ of etoposide for SKOV-3 cells was determined to be $1.9 \mu \mathrm{M}$ [24]. In order to assure that microorganisms would not be able to contaminate the SLNs and interfere with cytotoxicity results, preparation of solution of free drug and also preparation and dilution of SLNs suspensions were carried out in aseptic conditions under a laminar flow hood. It should be pointed out that solutions of organic and aqueous phases were presterilized by ultraviolet germicidal irradiation method.

Treated groups included either a solution of free drug in $1 \mathrm{w} / \mathrm{v} \%$ aqueous solution of Tween 80 or encapsulated drug in nontargeted and targeted nanoparticles, with blanks of nontargeted and targeted nanoparticles, while culture medium and Tween $801 \mathrm{w} / \mathrm{v} \%$ (each one in 8 wells) serve as control groups. The cells were incubated for further $48 \mathrm{~h}$. After the treatment, $20 \mu \mathrm{L} /$ well of the MTT solution ( $5 \mathrm{mg} / \mathrm{mL}$ of PBS) was added to the cells and incubated for $3 \mathrm{~h}$; then the supernatant was removed carefully and the formazan crystals were dissolved by adding $150 \mu \mathrm{L}$ of DMSO. Finally, the absorbance of each well was measured at $570 \mathrm{~nm}$ by an ELIZA plate reader (STAT FAX 2100 Microplate Reader, Awareness Technology, Inc., US). The effect of each treatment on cell viability was calculated by comparing the relative absorbance of treated cells against the respective controls, using the following equation [25]:

$$
\begin{aligned}
& \text { Cell survival \% } \\
& \begin{aligned}
= & \text { mean absorbance of each group } \\
& - \text { mean absorbance of blank }) \\
\times & (\text { mean absorbance of negative control } \\
& \quad-\text { mean absorbance of blank })^{-1} \\
& \times 100
\end{aligned}
\end{aligned}
$$

\subsection{Qualitative Comparison of Drug Uptake from Nanopar-} ticles by Fluorescence Imaging. First, $2700 \mu \mathrm{L}$ of the cellular suspension with the concentration of $10^{5}$ cells $/ \mathrm{mL}$ was poured into 10 wells of a 12 -well plate containing lamels at the bottom and then incubated for $48 \mathrm{~h}$ in $\mathrm{CO}_{2}$ incubator. Then the nontargeted and targeted nanoparticles were loaded with sodium fluorescein instead of etoposide by the same method as mentioned above for drug-loaded SLNs. The final concentration of loaded sodium fluorescein in nanoparticles was $1 \mathrm{mg} / \mathrm{mL}$. Blank nanoparticles were also prepared but without sodium fluorescein. To prepare free sodium fluorescein solution, $10 \mu \mathrm{L}$ of stock solution $(100 \mathrm{mg} / \mathrm{mL})$ was diluted to $1 \mathrm{~mL}$ to provide the final concentration of $1 \mathrm{mg} / \mathrm{mL}$.

Finally, $300 \mu \mathrm{L}$ of each sample was added to 2 wells (one for imaging in the 1st hour and the other for imaging in 4th hour) and was incubated. Lamels were withdrawn and imaging was performed by visible fluorescence microscope (Olympus, IX71, Japan) [11].

2.8. Statistical Analysis. All data are the results of three separate experiments, and the results are expressed as the mean \pm standard deviation $(n=3)$. Statistical analysis was performed using one-way analysis of variance (ANOVA) and an independent Student's $t$-test with the SPSS software (version 18, US). A $P$ value of less than 0.05 was considered significant. 
TABLE 1: Properties of solid lipid nanoparticles of etoposide.

\begin{tabular}{lccccc}
\hline SLNs type & Particle size $(\mathrm{nm})$ & pdI & zeta potential $(\mathrm{mV})$ & Drug loading efficiency $(\%)$ & RE in 24 h $(\%)$ \\
\hline Non-targeted SLNs & $179.6 \pm 16.3$ & $0.17 \pm 0.03$ & $11.82 \pm 0.52$ & - & - \\
HA targeted SLNs & $416.4 \pm 31.8$ & $0.30 \pm 0.05$ & $-12.65 \pm 0.49$ & $64.92 \pm 3.76$ & $65.47 \pm 4.68$ \\
\hline
\end{tabular}

\section{Results and Discussion}

3.1. Physicochemical Properties of Nanoparticles. Table 1 represents properties of nanoparticles. The particle size of nontargeted and targeted SLNs was $179.6 \pm 16.31$ and $416.42 \pm$ 31.85 , respectively. Zeta potential of nontargeted SLNs was $11.82 \pm 0.52$ that changed to $-12.65 \pm 0.49$ after coating with HA. Drug loading efficiency was about $64.92 \pm 3.76 \%$ and release efficiency percent in $24 \mathrm{~h}$ was $65.47 \pm 4.68 \%$ which is an acceptable value. HA was coated as much as 55.89 percent on the SLNs. Figure 2 represents drug release profile from HA targeted nanoparticles.

SLNs have generally long-term stability (about $1-3$ years) as small particle size and density close to unity of SLNs mean that the gravity has little effect on particles in dispersion and the Brownian motion is sufficient to maintain colloidal dispersions without creaming or sedimentation. In the present study the presence of physically bound HA and the negative zeta potential of targeted SLNs may seem to threaten stability, but our unpublished results showed that properties of the mentioned SLNs suspension did not change significantly within 10 days. However, as freeze-drying is a suitable method to prevent the Ostwald ripening and avoid aggregation of SLNs, we also dried the nontargeted and targeted SLNs under vacuum with 5\% glycerol serving as cryoprotectant and then recovered them by adding deionized water. The results showed that nontargeted SLNs only needed 5 minutes of stirring at $800 \mathrm{rpm}$ and targeted SLNs needed twice the stirring at $800 \mathrm{rpm}$ each time for 3 minutes and then 10 seconds of sonication at a power of $30 \mathrm{w}$, to retrieve their primary properties. Nonetheless, the SLNs which were used for cytotoxicity study were prepared fresh.

The observed release rate $(64.1 \%$ in the first 6 hours and $73.1 \%$ in 24 hours) could provide appropriate serum concentrations for routine chemotherapy schedules in which the drug (with an $i v$ half-life of 6-12 hours) is administered once daily. Also the mean diameter of typically 200-400 nm is well below the size of the smallest blood capillaries in the range of 5-6 $\mu \mathrm{m}$. Furthermore, because of the heterogeneity of tumors and dynamic status of each tumor, it will be very difficult to assume any maximum single value for particles to exploit the enhanced permeation and retention (EPR) effect. However, the study of Bae and Park suggests that the porosity of the blood vessels in tumors is around $400 \mathrm{~nm}$ [26]. A tumor-dependent functional pore cutoff size ranges from $200 \mathrm{~nm}$ to $1.2 \mu \mathrm{m}$, but the pore cutoff size of porous blood vessels in the majority of tumors is known to be $380-780 \mathrm{~nm}$ [27]. Thus, the range of the EPR effect should be similar. Sterically stabilized liposomes of $400 \mathrm{~nm}$ in diameter were able to penetrate into tumor interstitium [28]. Accumulation of hyaluronic acid-coated self-assembled nanoparticles with

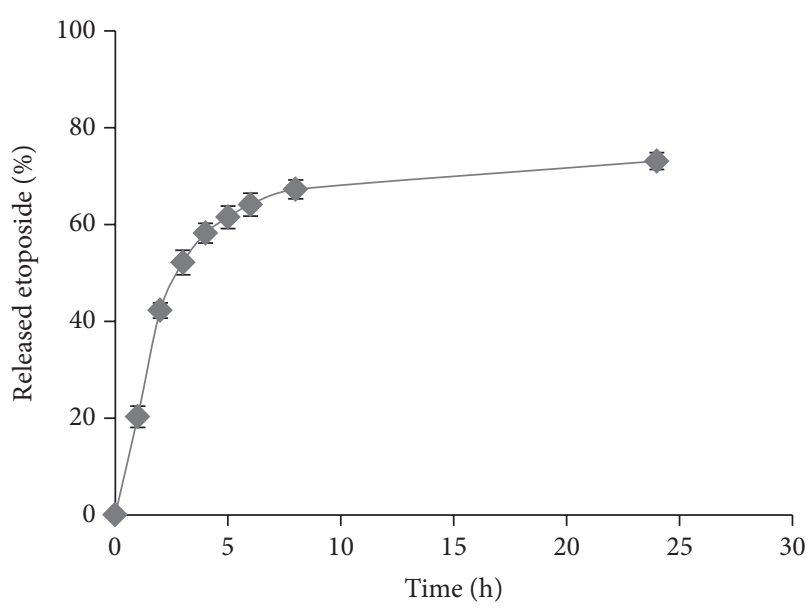

FIGURE 2: Etoposide release profile from HA targeted SLNs.

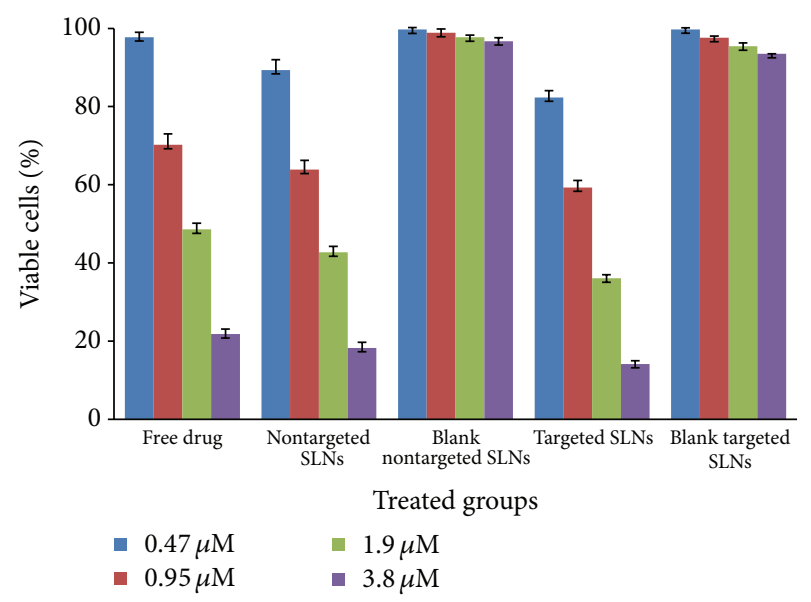

FIgURE 3: Percentage of viable cells of SK-OV-3 determined by the MTT assay after treatment with etoposide loaded nontargeted and hyaluronate targeted SLNs in comparison to blank nontargeted and targeted SLNs and free drug $(n=3)$.

particle size of $400 \mathrm{~nm}$ has been reported in the tumor tissue too [29].

3.2. Cell Proliferation Assay (MTT Assay). The obtained results of MTT cytotoxicity assay have been illustrated in Table 2 and Figure 3.

All drug-loaded nanoparticles caused higher cytotoxicity compared to the free etoposide at the same concentration and their respective blank SLNs. The mechanism of enhanced cytotoxicity of drug-loaded lipid nanoparticles 

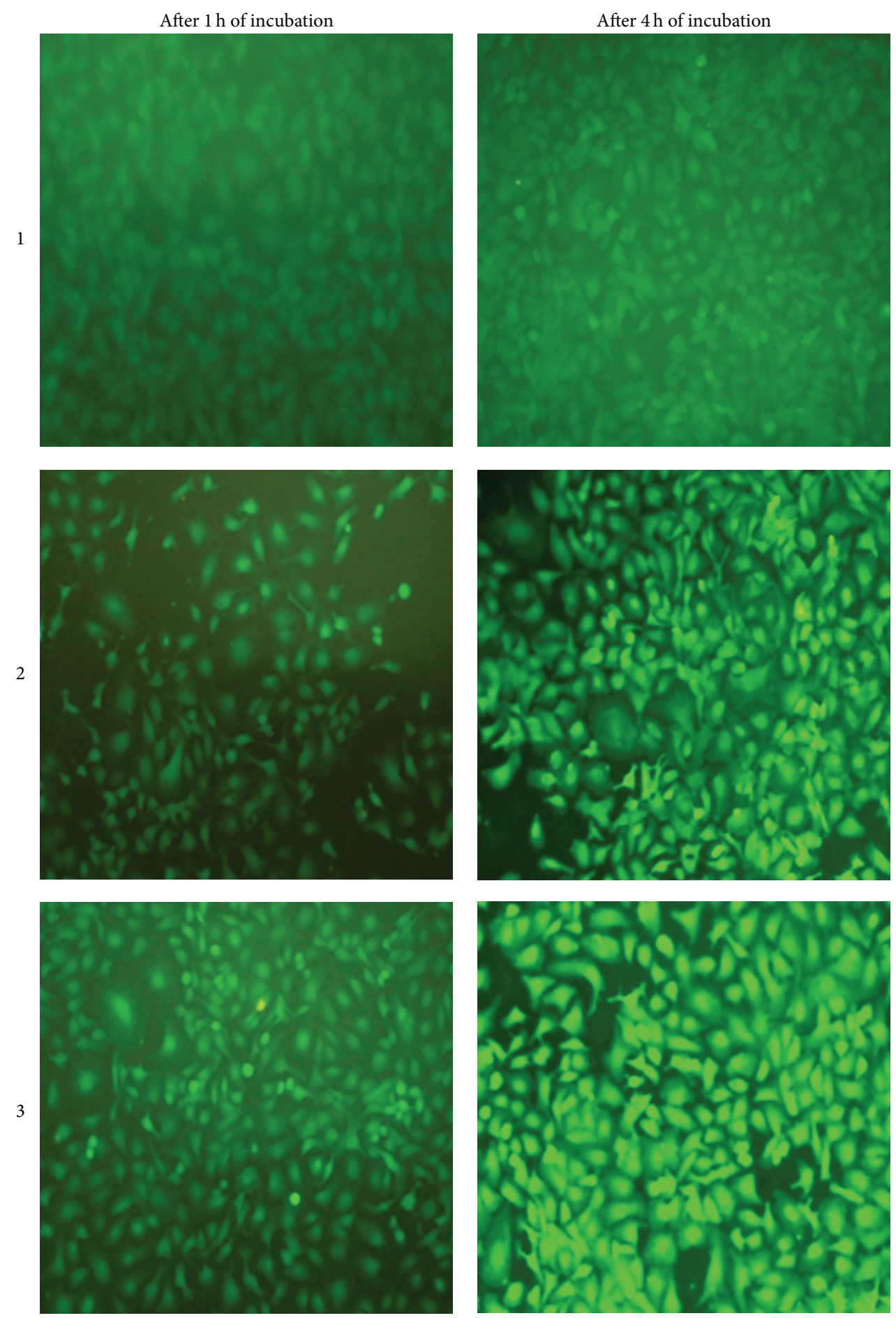

FIGURE 4: Fluorescence images of SK-OV-3 cells after 1 and 4 hours of incubation with (1) free sodium fluorescein, (2) sodium fluorescein containing nontargeted SLNs, and (3) sodium fluorescein containing targeted SLNs.

has been previously reported [30,31]. It is well understood that improvement in the cytotoxicity is because of the elevated drug concentrations within the cells. As we can see in Figure 3, nontargeted drug-loaded SLNs have lower cell survival compared to the free etoposide solution. For example, the observed cell survival after treatment with targeted nanoparticles was $36.08 \pm 0.88 \%$, while it was $42.73 \pm$ $1.49 \%$ and $48.57 \pm 1.61 \%$ for nontargeted SLNs and free drug solution, respectively, at the concentration of $1.9 \mu \mathrm{M}(P<$ 0.05).

The results verified that targeted and nontargeted SLNs of etoposide have reduced $\mathrm{IC}_{50}$ to $52 \%$ and $83 \%$ of free drug, 
TABLE 2: IC $_{50}$ of etoposide loaded in non-targeted and hyaluronate targeted SLNs in SK-OV-3 cells.

\begin{tabular}{lccc}
\hline & Free drug & Non-targeted SLNs & AH-targeted SLNs \\
\hline $\mathrm{IC}_{50}(\mu \mathrm{M})$ & $1.49 \pm 0.08$ & $1.24 \pm 0.13$ & $0.78 \pm 0.12$ \\
\hline
\end{tabular}

respectively (Table 2). In a study, Saliou et al. [32] reported that lipid nanocapsules of etoposide reduced the $\mathrm{IC}_{50}$ of the drug from 100 to $2.5 \mu \mathrm{M}$ in $\mathrm{H} 209$ cells. These lipid nanocapsules also could reduce the $\mathrm{IC}_{50}$ of etoposide to about 4-30 times in glioma cell lines [33]. In an experiment conducted by Nasti et al. [34] chitosan/triphosphate nanoparticles coated with $\mathrm{HA}$ showed the $\mathrm{IC}_{50}$ of about half of the noncoated nanoparticles on murine fibroblasts of L929 and macrophage cells of J774.2. Han et al. [35] successfully overcame on drug resistance of MCF-7/ADR cells with 4.3-fold reduction in $\mathrm{IC}_{50}$ of doxorubicin by SiRNA polyamidoamine-hyaluronic acid complex.

It could be concluded that the internalization of the drug into cells was enhanced when the drug was encapsulated in SLNs. This phenomenon might be the result of the high affinity of lipid materials of SLNs for the cell membrane and the nanoscaled size of SLNs.

The correlation between nanoparticles size and intracellular concentration has been observed in the study performed by Zhang et al. [36] and their results indicated that the less the particle size is, the more the intracellular drug concentration and cytotoxicity is.

In addition, comparing the targeted and nontargeted nanoparticles determines that the cytotoxicity in the targeted nanoparticles has been increased, probably due to the presence of HA on targeted nanoparticles which could interact with $\mathrm{CD}_{44}$ receptors and make them internalized into cells more easily. Cho et al. [37] have surveyed NPLs containing docetaxel targeted by HA upon cancer cell line MCF-7 and showed that they were endocytosed by $\mathrm{CD}_{44}$ receptors.

3.3. Cellular Uptake Studies. After incubating for 1 hour, only targeted nanoparticles made a slight fluorescence in the cells (Figure 4). After 4 hours of incubation, the fluorescence was remarkably higher in the cells which were treated by targeted SLNs rather than those which were treated by nontargeted nanoparticles, and there was no observable fluorescence in cells incubated with pure sodium fluorescein (Figure 4). Therefore, it could be concluded that increased cytotoxicity in results obtained from MTT assay has resulted from special uptake of targeted nanoparticles due to presence of HA as targeting agent.

\section{Conclusion}

Hyaluronate targeted SLNs containing etoposide increase the cytotoxicity of etoposide in SK-OV-3 cells and could be a valuable method for reducing the prescribed dose and also systemic side effects.

\section{Conflict of Interests}

The authors declare that there is no conflict of interests.

\section{Acknowledgment}

The authors would like to thank Vice Chancellor for Research of Isfahan University of Medical Sciences who provided project finances.

\section{References}

[1] A. E. Guppy, P. D. Nathan, and G. J. S. Rustin, "Epithelial ovarian cancer: a review of current management," Clinical Oncology, vol. 17, no. 6, pp. 399-411, 2005.

[2] P. J. O’Dwyer, B. Leyland-Jones, and M. T. Alonso, "Etoposide (VP-16-213). Current status of an active anticancer drug," New England Journal of Medicine, vol. 312, no. 11, pp. 692-700, 1985.

[3] R. E. Taylor, T. J. McElwain, A. Barrett, and M. J. Peckham, "Etoposide as a single agent in relapsed advanced lymphomas. A phase II study," Cancer Chemotherapy and Pharmacology, vol. 7, no. 2-3, pp. 175-177, 1982.

[4] S. Mukherjee, S. Ray, and R. S. Thakur, "Solid lipid nanoparticles: a modern formulation approach in drug delivery system," Indian Journal of Pharmaceutical Sciences, vol. 71, no. 4, pp. 349358, 2009.

[5] H. Li, X. Zhao, Y. Ma, G. Zhai, L. Li, and H. Lou, "Enhancement of gastrointestinal absorption of quercetin by solid lipid nanoparticles," Journal of Controlled Release, vol. 133, no. 3, pp. 238-244, 2009.

[6] M. Uner and G. Yener, "Importance of solid lipid nanoparticles (SLN) in various administration routes and future perspectives," International Journal of Nanomedicine, vol. 2, no. 3, pp. 289-300, 2007.

[7] W. Mehnert and K. Mäder, "Solid lipid nanoparticles: production, characterization and applications," Advanced Drug Delivery Reviews, vol. 47, no. 2-3, pp. 165-196, 2001.

[8] R. H. Müller, K. Mäder, and S. Gohla, "Solid lipid nanoparticles (SLN) for controlled drug delivery-a review of the state of the art," European Journal of Pharmaceutics and Biopharmaceutics, vol. 50, no. 1, pp. 161-177, 2000.

[9] K. S. Yadav, K. Chuttani, A. K. Mishra, and K. K. Sawant, "Long circulating nanoparticles of etoposide using PLGA-MPEG and PLGA-pluronic block copolymers: characterization, drugrelease, blood-clearance, and biodistribution studies," Drug Development Research, vol. 71, no. 4, pp. 228-239, 2010.

[10] L. H. Reddy, R. K. Sharma, K. Chuttani, A. K. Mishra, and R. R. Murthy, "Etoposide-incorporated tripalmitin nanoparticles with different surface charge: formulation, characterization, radiolabeling, and biodistribution studies," The AAPS Journal, vol. 6, no. 3, p. e23, 2004.

[11] B. A. Nevius, Y. P. Chen, J. L. Ferry, and A. W. Decho, "Surfacefunctionalization effects on uptake of fluorescent polystyrene nanoparticles by model biofilms," Ecotoxicology, vol. 21, no. 8, pp. 2205-2213, 2012.

[12] G. Kremser, T. Rath, B. Kunert et al., "Structural characterisation of alkyl amine-capped zinc sulphide nanoparticles," Journal of Colloid and Interface Science, vol. 369, no. 1, pp. 154-159, 2012.

[13] Y. Sun, Synthesis of gold-amine nanoparticles of various sizes using two different methods [M.S. thesis], Department of Chemistry, Kansas State University, Manhattan, Kan, USA, 2010. 
[14] Y. Luo, N. J. Bernshaw, Z.-R. Lu, J. Kopecek, and G. D. Prestwich, "Targeted delivery of doxorubicin by HPMA copolymerhyaluronan bioconjugates," Pharmaceutical Research, vol. 19, no. 4, pp. 396-402, 2002.

[15] K. Akima, H. Ito, Y. Iwata et al., "Evaluation of antitumor activities of hyaluronate binding antitumor drugs: synthesis, characterization and antitumor activity," Journal of Drug Targeting, vol. 4, no. 1, pp. 1-8, 1996.

[16] A. Rosato, A. Banzato, G. De Luca et al., "HYTAD1-p20: a new paclitaxel-hyaluronic acid hydrosoluble bioconjugate for treatment of superficial bladder cancer," Urologic Oncology: Seminars and Original Investigations, vol. 24, no. 3, pp. 207-215, 2006.

[17] D. Peer and R. Margalit, "Loading mitomycin C inside long circulating hyaluronan targeted nano-liposomes increases its antitumor activity in three mice tumor models," International Journal of Cancer, vol. 108, no. 5, pp. 780-789, 2004.

[18] H. Lee, H. Mok, S. Lee, Y. Oh, and T. G. Park, "Target-specific intracellular delivery of siRNA using degradable hyaluronic acid nanogels," Journal of Controlled Release, vol. 119, no. 2, pp. 245-252, 2007.

[19] Y. H. Yun, D. J. Goetz, P. Yellen, and W. Chen, "Hyaluronan microspheres for sustained gene delivery and site-specific targeting," Biomaterials, vol. 25, no. 1, pp. 147-157, 2004.

[20] J. Varshosaz, P. Mohammadi Ghalaei, and F. Hassanzadeh, "Hyaluronate targeted solid lipid nanoparticles of etoposide: optimization and in vitro characterization," Journal of Nanomaterials, vol. 2014, Article ID 345845, 12 pages, 2014.

[21] J. Varshosaz, S. Eskandari, and M. Tabakhian, "Production and optimization of valproic acid nanostructured lipid carriers by the Taguchi design," Pharmaceutical Development and Technology, vol. 15, no. 1, pp. 89-96, 2010.

[22] Y. Ataman-Önal, S. Munier, A. Ganée et al., "Surfactant-free anionic PLA nanoparticles coated with HIV-1 p24 protein induced enhanced cellular and humoral immune responses in various animal models," Journal of Controlled Release, vol. 112, no. 2, pp. 175-185, 2006.

[23] V. Teeranachaideekul, E. B. Souto, V. B. Junyaprasert, and R. H. Müller, "Cetyl palmitate-based NLC for topical delivery of Coenzyme Q10-development, physicochemical characterization and in vitro release studies," European Journal of Pharmaceutics and Biopharmaceutics, vol. 67, no. 1, pp. 141-148, 2007.

[24] H. Itamochi, T. Oishi, M. Shimada et al., "Inhibiting the mTOR pathway synergistically enhances cytotoxicity in ovarian cancer cells induced by etoposide through upregulation of c-Jun," Clinical Cancer Research, vol. 17, no. 14, pp. 4742-4750, 2011.

[25] W. Strober, Monitoring Cell Growth. Current Protocols InImmunology, John Wiley \& Sons, Washington, DC, USA, 2001.

[26] Y. H. Bae and K. Park, "Targeted drug delivery to tumors: myths, reality and possibility," Journal of Controlled Release, vol. 153, no. 3, pp. 198-205, 2011.

[27] S. K. Hobbs, W. L. Monsky, F. Yuan et al., "Regulation of transport pathways in tumor vessels: role of tumor type and microenvironment," Proceedings of the National Academy of Sciences of the United States of America, vol. 95, no. 8, pp. 46074612, 1998.

[28] F. Yuan, M. Dellian, D. Fukumura et al., "Vascular permeability in a human tumor xenograft: molecular size dependence and cutoff size," Cancer Research, vol. 55, no. 17, pp. 3752-3756, 1995.

[29] K. Y. Choi, K. H. Min, J. H. Na et al., "Self-assembled hyaluronic acid nanoparticles as a potential drug carrier for cancer therapy: synthesis, characterization, and in vivo biodistribution," Journal of Materials Chemistry, vol. 19, no. 24, pp. 4102-4107, 2009.

[30] A. Miglietta, R. Cavalli, C. Bocca, L. Gabriel, and M. Rosa Gasco, "Cellular uptake and cytotoxicity of solid lipid nanospheres (SLN) incorporating doxorubicin or paclitaxel," International Journal of Pharmaceutics, vol. 210, no. 1-2, pp. 61-67, 2000.

[31] L. Serpe, M. G. Catalano, R. Cavalli et al., "Cytotoxicity of anticancer drugs incorporated in solid lipid nanoparticles on HT-29 colorectal cancer cell line," European Journal of Pharmaceutics and Biopharmaceutics, vol. 58, no. 3, pp. 673-680, 2004.

[32] B. Saliou, O. Thomas, N. Lautram et al., "Development and in vitro evaluation of a novel lipid nanocapsule formulation of etoposide," European Journal of Pharmaceutical Sciences, vol. 50, no. 2, pp. 172-180, 2013.

[33] A. Lamprecht and J. Benoit, "Etoposide nanocarriers suppress glioma cell growth by intracellular drug delivery and simultaneous P-glycoprotein inhibition," Journal of Controlled Release, vol. 112, no. 2, pp. 208-213, 2006.

[34] A. Nasti, N. M. Zaki, P. De Leonardis et al., "Chitosan/TPP and chitosan/TPP-hyaluronic acid nanoparticles: systematic optimisation of the preparative process and preliminary biological evaluation," Pharmaceutical Research, vol. 26, no. 8, pp. 19181930, 2009

[35] M. Han, Q. Lv, X. J. Tang et al., "Overcoming drug resistance of MCF-7/ADR cells by altering intracellular distribution of doxorubicin via MVP knockdown with a novel siRNA polyamidoamine-hyaluronic acid complex," Journal of Controlled Release, vol. 163, no. 2, pp. 136-144, 2012.

[36] X. G. Zhang, J. Miao, Y. Q. Dai, Y. Z. Du, H. Yuan, and F. Q. Hu, "Reversal activity of nanostructured lipid carriers loading cytotoxic drug in multi-drug resistant cancer cells," International Journal of Pharmaceutics, vol. 361, no. 1-2, pp. 239244, 2008.

[37] H. J. Cho, H. Y. Yoon, H. Koo et al., "Self-assembled nanoparticles based on hyaluronic acid-ceramide (HA-CE) and Pluronic $\AA$ for tumor-targeted delivery of docetaxel," Biomaterials, vol. 32, no. 29, pp. 7181-7190, 2011. 

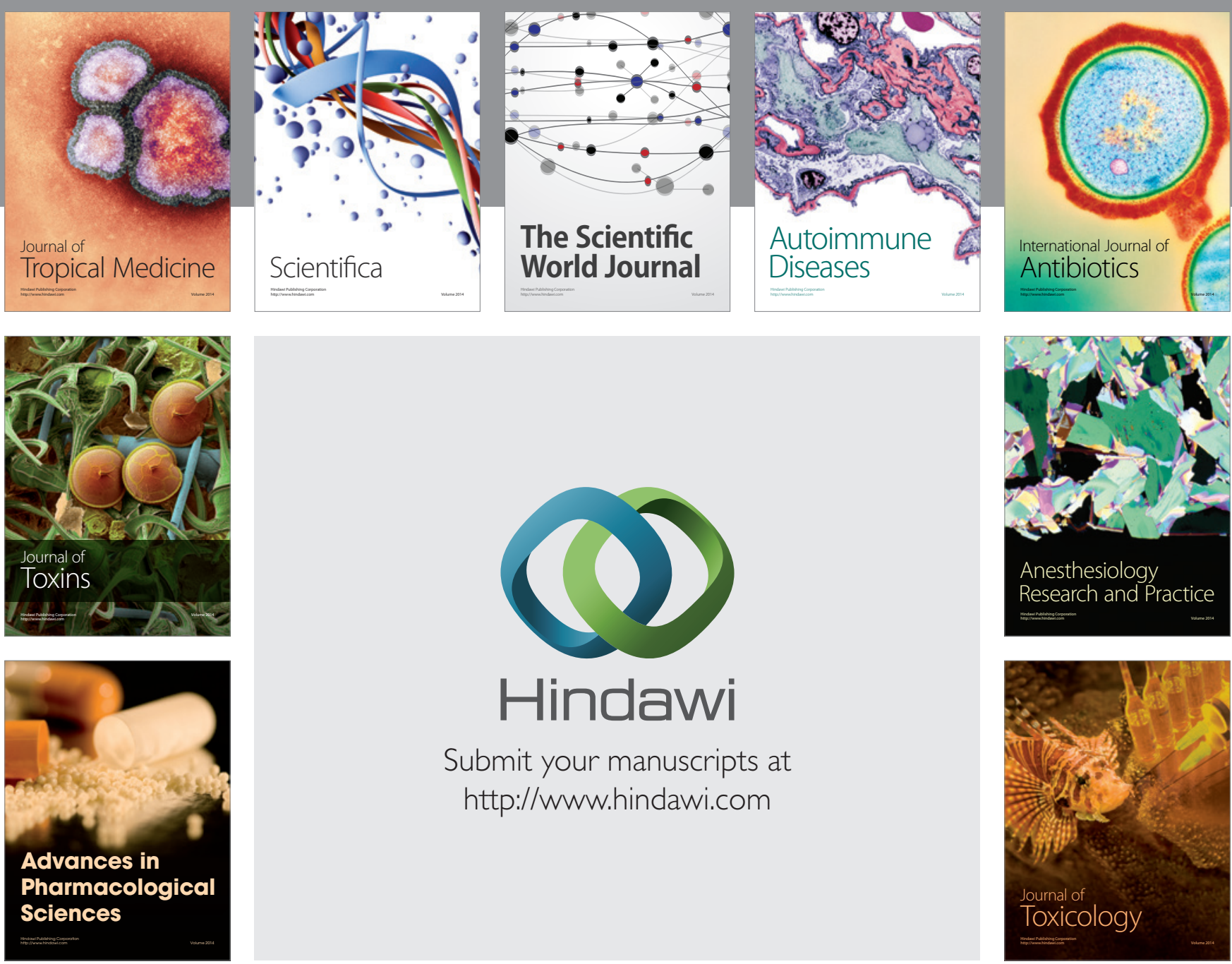

\section{Hindawi}

Submit your manuscripts at

http://www.hindawi.com
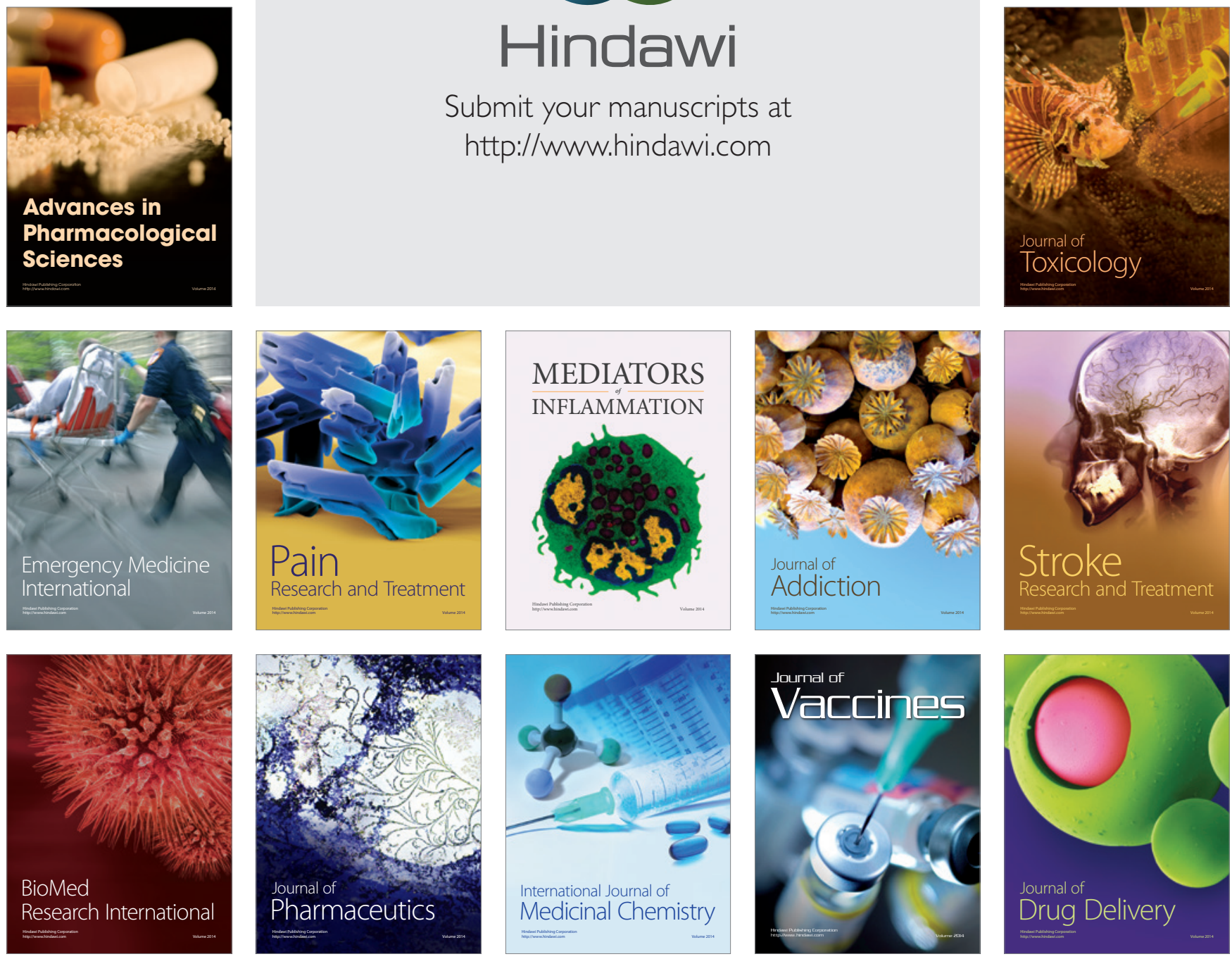